\title{
IL-10 and PRKDC polymorphisms are associated with glioma patient survival
}

\author{
Mingjun Hu${ }^{1,2}$, Jieli Du ${ }^{3,4}$, Lihong Cui ${ }^{5}$, Tingqin Huang ${ }^{1}$, Xiaoye Guo ${ }^{1}$, Yonglin Zhao ${ }^{1}$, \\ Xudong $\mathrm{Ma}^{1}$, Tianbo Jin' ${ }^{6}$, Gang Li ${ }^{7}$, Jinning Song ${ }^{1}$ \\ ${ }^{1}$ Department of Neurosurgery, First Affiliated Hospital of Medical College, Xi'an Jiaotong University, Xi'an 710061, China \\ ${ }^{2}$ Department of Neurosurgery, Xi'an First Hospital, Xi'an 710002, China \\ ${ }^{3}$ Inner Mongolia Medical University, Hohhot, Inner Mongolia, 010050, China \\ ${ }^{4}$ Department of Orthopedics and Traumatology, The 2nd Affiliated Hospital of Inner Mongolia University, Hohhot, Inner \\ Mongolia, 010030, China \\ ${ }^{5}$ Department of Neurology, Shangluo Central Hospital, Shangluo 726000, China \\ ${ }^{6}$ Key Laboratory of Resource Biology and Biotechnology in Western China, Northwest University, Ministry of Education, \\ School of Life Sciences, Northwest University, Xi'an 710069, China \\ ${ }^{7}$ Department of Neurosurgery, Tangdu Hospital, The Fourth Military Medical University, Xi'an 710038, China
}

Correspondence to: Jinning Song, email: jinningsong@126.com

Keywords: IL-10, PRKDC, biomarker, glioma, polymorphism

Received: August 23, $2016 \quad$ Accepted: October 24, $2016 \quad$ Published: November 02, 2016

\section{ABSTRACT}

Interleukin-10 (IL-10) and DNA repair gene PRKDC mutations are implicated in the development of multiple human cancers, including glioma. We investigated associations between $I L-10$ and PRKDC gene polymorphisms and prognosis in lowand high-grade glioma patients. We analyzed the associations of one $I L-10$ and one PRKDC single nucleotide polymorphism with patient clinical factors in $\mathbf{4 8 1}$ glioma patients using Cox proportional hazard models and Kaplan-Meier curves. We also assessed associations between patient clinical characteristics and prognosis. Our data showed that the extent of tumor resection (gross-total resection) and application of chemotherapy were associated with improved patient outcomes in all glioma cases. Additionally, univariate (Log-rank $p=0.019)$ and multivariate Cox regression analyses $(p=0.022)$ showed that the $I L-10$ rs $1800871 \mathrm{C} / \mathrm{T}$ genotype correlates with improved overall survival in cases of low-grade glioma, whereas the PRKDC rs7003908 C/C genotype correlated with reduced overall and progression-free survival in high-grade glioma patients in univariate (Log-rank $p=0.000$ and $p=0.000$, respectively) and multivariate Cox regression analyses $(p=0.001 ; p=0.002$, respectively). These results suggest that $I L-10$ rs1800871 and PRKDC rs7003908 may be useful biomarkers for predicting glioma patient outcome. Further functional studies are needed to evaluate the mechanisms by which these polymorphisms affect glioma progression.

\section{INTRODUCTION}

Glioma is a general term used to describe any tumor that arises from the supportive (gluey) tissue of the brain. This tissue, called glia, helps to keep the neurons in place and functioning well. It is the most common and aggressive primary brain tumors, occurring most commonly in adults and accounting for $40-50 \%$ of all brain tumors [1]. According to the 2007 World Health Organization (WHO) classification, gliomas are subclassified into four grades based on degree of aggressiveness: grade I (pilocytic astrocytomas), grade II (diffuse infiltrating lowgrade gliomas), grade III (anaplastic gliomas), and grade IV (glioblastomas multiforme) [2]. Low-grade gliomas (grade I-II) are well differentiated, and generally have better prognoses than high-grade gliomas (grade III-IV) [2, 3]. Despite total or near-total tumor resection, and advancements in chemo-radiotherapy, patient outcomes remain dismal. Median survival times for patients with grade III and IV gliomas are only 39 and 30 weeks, respectively [4]. 
Several clinical factors, such as surgical method, radiotherapy, chemotherapy, patient age and gender, and tumor grade, size, range and location, can affect glioma patient prognosis. Most gliomas result from the combined action of environmental factors and inherited genetic variations [5]. Haque, et al. [6] suggested that glioblastoma arises from genetic and epigenetic alterations in normal astroglial cells, implying that genetic factors are primarily responsible for gliomagenesis.

Interleukin-10 (IL-10), an important antiinflammatory cytokine, is secreted by various immune cells. IL-10 has pleiotropic effects on immunoregulation and inflammation, and can promote carcinogenesis [7]. Huettner, et al. [8] suggested that IL-10 promotes glioma progression by enhancing tumor cell invasion and providing an immunosuppressive environment. Additionally, $I L-10$ polymorphisms are associated with glioblastoma [9].

$P R K D C$, also known as human X-ray repair cross-complementing group 7 (XRCC7), is located on chromosome 8q11. Several studies have associated $P R K D C$ with poor prognosis in numerous tumor types, such as esophageal cancer [10], B-cell chronic lymphocytic leukemias [11], and colorectal cancer [12]. PRKDC gene polymorphisms are also correlated with glioma risk $[13,14]$.

Although previous association studies linked $I L-10$ and $P R K D C$ genetic polymorphisms with glioma risk, few focused on the effects of these alterations on glioma patient prognosis. In the present study, we selected one single nucleotide polymorphism (SNP) in $I L-10$ [9] and one in PRKDC [14], each of which was reportedly associated with glioma risk. We evaluated the associations between these two SNPs and low- and high-grade glioma patient prognosis in a Chinese population.

\section{RESULTS}

\section{Patient clinical data}

A total of 481 glioma patients were analyzed in this study, including 300 astrocytomas, 70 oligodendrocytomas, 43 glioblastomas, 31 ependymomas, 14 oligodendrogliomas, and 23 other types of gliomas. Detailed clinical data for glioma patients are summarized in Table 1. Patients included $264(54.9 \%)$ men and 217 (45.1\%) women, with 208 patients $<40$ years of age and $273 \geq 40$ years of age. A total of $35(7.3 \%)$ patients were classified as WHO grade I, $259(53.8 \%)$ as WHO grade II, 124 $(25.8 \%)$ as WHO grade III and $63(13.1 \%)$ as WHO grade IV. $328(68.2 \%)$ patients received gross-total tumor surgical resections and $153(31.3 \%)$ received near-total or sub-total resections. Gamma knife radiotherapy was administered to $315(65.5 \%)$ patients and conformal radiation therapy was administered to 122 (25.4\%). Finally, 198 (41.1\%) patients received chemotherapy. 438 (91.1\%) patients had died at the last follow-up and 453 (94.2\%) progressed during follow-up.

\section{Univariate analysis}

In a univariate analysis of clinical factors, including patient gender and age, extent of tumor resection, and radiotherapy and chemotherapy regimen, we found that the median overall survival (OS) of WHO grade I-II glioma patients (12 months; 3-year survival rate $=9.0 \%$ ) was longer than that of WHO grade III-IV patients (10 months; 3-year survival rate $=5.5 \%)(\log -\operatorname{rank} p=0.039)$ (Tables 2-4). The extent of resection (gross-total resection) was a protective factor, as was chemotherapy, in all glioma patients. Overall, gross-total resection was associated with a $33.3($ Log-rank $p=0.000, \mathrm{HR}=0.667$, $95 \% \mathrm{CI}=0.545-0.817, p=0.000)$ and $36.5 \%$ (Log-rank $p=0.000, \mathrm{HR}=0.63 .5,95 \% \mathrm{CI}=0.519-0.777, p=0.000)$ decrease in OS and PFS mortality hazards, respectively. Median OS and PFS were 11 and 8 months, respectively, with $10.2 \%$ and $6.0 \% 3$-year survival rates. In low-grade glioma patients, gross-total resection was associated with a $32.3 \%($ Log-rank $p=0.000, \mathrm{HR}=0.677,95 \%$ $\mathrm{CI}=0.522-0.878, p=0.003$ ) and $35.8 \%$ (Log-rank $p=0.000, \mathrm{HR}=64.2,95 \% \mathrm{CI}=0.496-0.831, p=0.001)$ decrease in OS and PFS mortality hazards, respectively. Median OS and PFS were 11 and 8 months, respectively, with $12.5 \%$ and $7.1 \% 3$-year survival rates. Finaly, in highgrade glioma patients, gross-total resection was associated with a $37.6 \%($ Log-rank $p=0.002, \mathrm{HR}=0.62 .4,95 \%$ $\mathrm{CI}=0.451-0.864, p=0.004)$ and $39.3 \%$ (Log-rank $p$ $=0.001, \mathrm{HR}=60.7,95 \% \mathrm{CI}=0.439-0.840, p=0.003$ ) decrease in OS and PFS mortality hazard, respectively. Median OS and PFS were 10 and 8 months, respectively, with $6.9 \%$ and $4.5 \% 3$-year survival rates. Treatment with chemotherapy was also associated with an overall reduced risk of death as measured by increased patient OS (3-year survival rate $=14.2 \%$, median survival $=12$ months, Log-rank $p=0.000, \mathrm{HR}=0.643,95 \% \mathrm{CI}=0.528-0.782$, $p=0.000)$ and PFS (3-years survival rate $=11.6 \%$, median survival $=8$ months, Log-rank $p=0.000, \mathrm{HR}=0.731$, $95 \% \mathrm{CI}=0.602-0.889, p=0.002)$. This same trend was observed in OS (3-year survival rate $=16.2 \%$, median survival $=12$ months, Log-rank $p=0.000, \mathrm{HR}=0.665$, $95 \%$ CI $=0.517-0.855, p=0.001)$ and PFS (3-year survival rate $=13.3 \%$, median survival $=8$ months, Logrank $p=0.004, \mathrm{HR}=0.722,95 \% \mathrm{CI}=0.523-0.926$, $p=0.010$ ) of low-grade glioma patients, and in OS (3-year survival rate $=11.3 \%$, median survival $=12$ months, Log-rank $p=0.001, \mathrm{HR}=0.617,95 \% \mathrm{CI}=0.450-0.846$, $p=0.003$ ) of high-grade patients. No significant correlations were identified between patient gender and age, WHO tumor grade, or radiotherapy and prognosis as measured by OS and PFS.

According to Log-rank tests and Cox regression analysis, the two SNPs we studied were not correlated with OS or PFS in glioma patients (Table 5). However, we found that the $I L-10 \mathrm{rs} 1800871 \mathrm{C} / \mathrm{T}$ genotype was associated with increased OS in low-grade glioma cases (3- 
Table 1: Characteristics of glioma patients

\begin{tabular}{|c|c|c|c|c|c|c|c|}
\hline \multirow[b]{2}{*}{ Variable } & \multirow[b]{2}{*}{ Classification } & \multicolumn{2}{|c|}{ Glioma } & \multicolumn{2}{|c|}{ I-II } & \multicolumn{2}{|c|}{ III-IV } \\
\hline & & No. of Patients & Percent \% & $\begin{array}{c}\text { No. of } \\
\text { Patients }\end{array}$ & Percent \% & No. of Patients & Percent \% \\
\hline \multirow[t]{2}{*}{ Gender } & Male & 264 & 54.9 & 157 & 53.4 & 107 & 57.2 \\
\hline & Female & 217 & 45.1 & 137 & 46.6 & 80 & 42.8 \\
\hline \multirow[t]{2}{*}{ Age(years) } & $<40$ & 208 & 43.2 & 144 & 49.0 & 64 & 34.2 \\
\hline & $\geq 40$ & 273 & 56.8 & 150 & 51.0 & 123 & 65.8 \\
\hline \multirow[t]{4}{*}{ WHO grade } & WHO I & 35 & 7.3 & 35 & 11.9 & & \\
\hline & WHO II & 259 & 53.8 & 259 & 88.1 & & \\
\hline & WHO III & 124 & 25.8 & & & 124 & 66.3 \\
\hline & WHO IV & 63 & 13.1 & & & 63 & 33.7 \\
\hline \multirow[t]{2}{*}{ Extent of resection } & GTR & 328 & 68.2 & 197 & 67.0 & 131 & 70.1 \\
\hline & STR or NTR & 153 & 31.8 & 97 & 33.0 & 56 & 29.9 \\
\hline \multirow[t]{3}{*}{ Radiotherapy } & GK & 315 & 65.5 & 197 & 67.0 & 118 & 63.1 \\
\hline & CRT & 122 & 25.4 & 73 & 24.8 & 49 & 26.2 \\
\hline & No & 44 & 9.1 & 24 & 8.2 & 20 & 10.7 \\
\hline \multirow[t]{4}{*}{ Chemotherapy } & Platinum & 101 & 21.0 & 63 & 21.4 & 38 & 20.3 \\
\hline & Nimustine & 60 & 12.5 & 35 & 11.9 & 25 & 13.4 \\
\hline & Temozolomide & 37 & 7.7 & 26 & 8.8 & 11 & 5.9 \\
\hline & No & 283 & 58.8 & 170 & 57.8 & 113 & 60.4 \\
\hline \multirow[t]{3}{*}{ Survival condition } & Survival & 26 & 5.4 & 17 & 5.8 & 9 & 4.8 \\
\hline & Lost & 17 & 3.5 & 14 & 4.8 & 3 & 1.6 \\
\hline & Death & 438 & 91.1 & 263 & 89.5 & 175 & 93.6 \\
\hline \multirow[t]{3}{*}{ Progress } & Yes & 453 & 94.2 & 275 & 93.5 & 178 & 95.2 \\
\hline & No & 24 & 5.0 & 17 & 5.8 & 7 & 3.7 \\
\hline & Missing system & 4 & 0.8 & 2 & 0.7 & 2 & 1.1 \\
\hline
\end{tabular}

WHO: World Health Organization; GTR: Gross-total resection; NTR: Near-total resection; STR: Sub-total resection; GK: Gamma knife; CRT: Conformal radiation therapy.

year survival rate $=13.1 \%$, median survival $=12$ months, Log-rank $p=0.019, \mathrm{HR}=0.745,95 \% \mathrm{CI}=0.573-0.969$, $p=0.028$, but not high-grade cases. We also found that the PRKDC rs7003908 C/C genotype correlated with poor prognosis in high-grade glioma patients as measured by OS (3-year survival rate $=0.0 \%$, median survival $=6$ months, Log-rank $p=0.000, \mathrm{HR}=4.556$, 95\% CI $=1.936-10.721, p=0.001)$ and PFS (3-year survival rate $=0.0 \%$, median survival $=4$ months, Log-rank $p=0.000, \mathrm{HR}=4.430,95 \%$ CI $=1.884-10.416$, $p=0.001)$, although this was not observed in low-grade glioma patients.

\section{Multivariate analysis}

Multivariate Cox regression analysis demonstrated that SNP genotype was an independent prognostic factor for OS and PFS after adjustment for the various clinical factors. In the present study, we identified correlations between rs1800871, rs7003908 and prognosis in different glioma grades (Table 6). The $I L-10 \mathrm{rs} 1800871 \mathrm{C} / \mathrm{T}$ genotype correlated with better OS in low-grade glioma patients (adjusted HR $=0.736,95 \% \mathrm{CI}=0.565-0.958$, $p=0.022$ ) and the PRKDC rs7003908 C/C genotype correlated with worse OS (adjusted HR $=4.288,95 \%$ $\mathrm{CI}=1.808-10.169, p=0.001$ ) and PFS (adjusted $\mathrm{HR}=3.783,95 \% \mathrm{CI}=1.601-8.943, p=0.002)$ in highgrade glioma patients.

\section{DISCUSSION}

Gliomas are the most common primary tumors of the central nervous system and several patient prognosis predictors have been identified, including patient age, WHO tumor grade, extent of tumor resection, and radiotherapy and chemotherapy regimen(s) [15]. Consistent with previous studies [16, 17], we found that chemotherapy and the extent of resection were key prognostic factors in glioma patients. Prognoses differed between low-grade and high-grade glioma patients. After WHO grade stratification, we found that rs1800871 in $I L-10$ correlated with increased OS in low-grade glioma 
Table 2: Univariate analysis of the impact of clinical factors on glioma patient OS and PFS

\begin{tabular}{|c|c|c|c|c|c|c|c|c|c|c|c|c|c|}
\hline \multirow{2}{*}{ Variable } & \multirow{2}{*}{ Classification } & \multirow{2}{*}{$\begin{array}{c}\text { No. of } \\
\text { patients/ } \\
\text { events }\end{array}$} & \multicolumn{4}{|c|}{ OS } & \multirow[b]{2}{*}{$p$} & \multirow{2}{*}{$\begin{array}{c}\text { No. of } \\
\text { patients/ } \\
\text { events }\end{array}$} & \multicolumn{5}{|c|}{ PFS } \\
\hline & & & $\begin{array}{c}\text { 1/3-(year) } \\
\text { SR (\%) }\end{array}$ & MST & Log-rank $p$ & HR $(95 \% \mathrm{CI})$ & & & $\begin{array}{c}\text { 1/3-(year) } \\
\text { SR (\%) }\end{array}$ & MST & Log-rank $p$ & HR $(95 \% \mathrm{CI})$ & $p$ \\
\hline \multirow[t]{2}{*}{ Gender } & Male & $264 / 241$ & $30.3 / 7.0$ & 11 & & 1 & & $262 / 248$ & $17.6 / 4.9$ & 8 & & 1 & \\
\hline & Female & $217 / 197$ & $31.8 / 8.2$ & 10 & 0.583 & $1.049(0.869-1.267)$ & 0.616 & $215 / 205$ & $13.5 / 4.3$ & 8 & 0.565 & $1.050(0.872-1.263)$ & 0.607 \\
\hline \multirow[t]{2}{*}{ Age (years) } & $<40$ & $208 / 186$ & $33.2 / 9.1$ & 12 & & 1 & & $205 / 191$ & $17.1 / 6.1$ & 8 & & 1 & \\
\hline & $\geq 40$ & $273 / 252$ & $29.3 / 6.3$ & 11 & 0.202 & $1.119 /(0.926-1.353)$ & 0.244 & $272 / 262$ & $14.7 / 3.5$ & 8 & 0.135 & $1.136(0.942-1.370)$ & 0.182 \\
\hline \multirow[t]{2}{*}{ WHO grade } & I-II & $294 / 263$ & $33.0 / 9.0$ & 12 & & 1 & & $292 / 275$ & $17.1 / 5.4$ & 8 & & 1 & \\
\hline & III-IV & $187 / 175$ & $27.8 / 5.5$ & 10 & 0.039 & $1.202(0.993-1.456)$ & 0.059 & $185 / 178$ & $13.5 / 3.7$ & 8 & 0.119 & $1.144(0.947-1.381)$ & 0.163 \\
\hline \multirow{2}{*}{$\begin{array}{l}\text { Extent of } \\
\text { resection }\end{array}$} & STR or NTR & $153 / 150$ & $19.6 / 2.0$ & 12 & & 1 & & $150 / 147$ & $2.0 / 2.0$ & 8 & & 1 & \\
\hline & GTR & $328 / 288$ & $36.3 / 10.2$ & 11 & 0.000 & $0.667(0.545-0.817)$ & 0.000 & $327 / 306$ & $22.0 / 6.0$ & 8 & 0.000 & $0.635(0.519-0.777)$ & 0.000 \\
\hline \multirow[t]{3}{*}{ Radiotherapy } & No & $44 / 37$ & $40.9 / 15.9$ & 8 & & 1 & & $41 / 41$ & $17.1 / 2.4$ & 10 & & 1 & \\
\hline & CRT & $122 / 104$ & $21.3 / 12.9$ & 9 & & $1.089(0.747-1.589)$ & 0.657 & $121 / 107$ & $15.7 / 11.4$ & 7 & & $1.195(0.830-1.720)$ & 0.338 \\
\hline & GK & $315 / 297$ & $33.3 / 5.3$ & 11 & 0.846 & $1.096(0.778-1.544)$ & 0.599 & $315 / 305$ & $15.6 / 2.6$ & 8 & 0.543 & $1.108(0.798-1.537)$ & 0.54 \\
\hline \multirow[t]{2}{*}{ Chemotherapy } & No & $283 / 270$ & $24.7 / 0.0$ & 9 & & 1 & & $282 / 282$ & $14.9 / 0.0$ & 7 & & 1 & \\
\hline & Yes & $198 / 168$ & $39.9 / 14.2$ & 12 & 0.000 & $0.643(0.528-0.782)$ & 0.000 & $195 / 171$ & $16.9 / 11.6$ & 8 & 0.000 & $0.731(0.602-0.889)$ & 0.002 \\
\hline
\end{tabular}

OS: Overall survival; PFS: Progression free survival; WHO: World Health Organization; GTR: Gross-total resection; NTR: Near-total resection;

STR: Sub-total resection; CRT: Conformal radiation therapy; GK: Gamma knife; SR: Survival rate; MST: Median survival time(months).

HR: Hazard ratio; $95 \%$ CI: $95 \%$ Confidence interval.

Log-rank p values were calculated from Chi-Square test.

$p$ values were calculated from Wald test.

$p<0.05$ indicates statistical significance.

Table 3: Univariate analysis of the impact of clinical factors on low-grade glioma patient OS and PFS

\begin{tabular}{|c|c|c|c|c|c|c|c|c|c|c|c|c|c|}
\hline \multirow[b]{2}{*}{ Variable } & \multirow[b]{2}{*}{ Classification } & \multirow{2}{*}{$\begin{array}{c}\text { No. of } \\
\text { patients/ } \\
\text { events }\end{array}$} & \multicolumn{4}{|c|}{ OS } & \multirow[b]{2}{*}{$p$} & \multirow{2}{*}{$\begin{array}{c}\text { No. of } \\
\text { patients/ } \\
\text { events }\end{array}$} & \multicolumn{5}{|c|}{ PFS } \\
\hline & & & $\begin{array}{c}\text { 1/3-(year) } \\
\text { SR (\%) }\end{array}$ & MST & Log-rank $p$ & HR (95\% CI) & & & $\begin{array}{c}\text { 1/3-(year) } \\
\text { SR (\%) }\end{array}$ & MST & Log-rank $p$ & HR $(95 \%$ CI $)$ & $p$ \\
\hline \multirow[t]{2}{*}{ Gender } & Male & $157 / 139$ & $35.0 / 9.8$ & 12 & & 1 & & $156 / 143$ & $21.8 / 7.9$ & 8 & & 1 & \\
\hline & Female & $137 / 124$ & $30.7 / 8.4$ & 12 & 0.241 & $1.142(0.895-1.456)$ & 0.285 & $136 / 132$ & $11.8 / 2.5$ & 8 & 0.097 & $1.197(0.944-1.518)$ & 0.137 \\
\hline \multirow[t]{2}{*}{ Age (years) } & $<40$ & $144 / 127$ & $33.3 / 10.4$ & 12 & & 1 & & $142 / 131$ & $17.6 / 6.7$ & 8 & & 1 & \\
\hline & $\geq 40$ & $150 / 136$ & $32.7 / 7.7$ & 11 & 0.487 & $1.082(0.849-1.378)$ & 0.526 & $150 / 144$ & $16.7 / 3.9$ & 8 & 0.325 & $1.113(0.878-1.411)$ & 0.377 \\
\hline \multirow[t]{3}{*}{ WHO grade } & I & $35 / 30$ & $31.4 / 12.0$ & 9 & & 1 & & $35 / 34$ & $20.0 / 2.9$ & 6 & & 1 & \\
\hline & II & $259 / 233$ & $33.2 / 8.7$ & 12 & 0.792 & $0.954(0.652-1.396)$ & 0.810 & $257 / 241$ & $16.7 / 5.8$ & 8 & 0.413 & $0.847(0.610-1.252)$ & 0.462 \\
\hline & STR or NTR & $97 / 95$ & $21.6 / 2.1$ & 12 & & 1 & & $95 / 93$ & $2.1 / 2.1$ & 8 & & 1 & \\
\hline Extent of resection & GTR & $197 / 168$ & $38.6 / 12.5$ & 11 & 0.001 & $0.677(0.522-0.878)$ & 0.003 & $197 / 182$ & $24.4 / 7.1$ & 8 & 0.000 & $0.642(0.496-0.831)$ & 0.001 \\
\hline \multirow[t]{3}{*}{ Radiotherapy } & No & $24 / 19$ & $45.8 / 20.8$ & 12 & & 1 & & $22 / 22$ & $22.7 / 0.0$ & 10 & & 1 & \\
\hline & CRT & $73 / 62$ & $20.5 / 13.2$ & 12 & & $1.270(0.758-2.128)$ & 0.363 & $73 / 65$ & $13.7 / 10.8$ & 8 & & $1.373(0.843-2.237)$ & 0.202 \\
\hline & GK & $197 / 182$ & $36.0 / 6.8$ & 12 & 0.605 & $1.196(0.745-1.920)$ & 0.459 & $197 / 188$ & $17.8 / 3.8$ & 8 & 0.299 & $1.176(0.754-1.832)$ & 0.474 \\
\hline \multirow[t]{2}{*}{ Chemotherapy } & No & $170 / 160$ & $28.2 / 0.0$ & 10 & & 1 & & $170 / 170$ & $16.5 / 0.0$ & 8 & & 1 & \\
\hline & Yes & $124 / 103$ & $39.5 / 16.2$ & 12 & 0.000 & $0.665(0.517-0.855)$ & 0.001 & $122 / 105$ & $18.0 / 13.3$ & 8 & 0.004 & $0.722(0.563-0.926)$ & 0.010 \\
\hline
\end{tabular}

OS: Overall survival; PFS: Progression free survival; WHO: World Health Organization; GTR: Gross-total resection; NTR: Near-total resection.

STR: Sub-total resection; CRT: Conformal radiation therapy; GK: Gamma knife; SR: Survival rate; MST: Median survival time (months).

HR: Hazard ratio; $95 \%$ CI: $95 \%$ Confidence interval.

Log-rank $\mathrm{p}$ values were calculated from Chi-Square test.

$p$ values were calculated from Wald test.

$p<0.05$ indicates statistical significance.

cases, while rs 7003908 in $P R K D C$ correlated with poor prognosis in high-grade cases.

Ours was the first study to associate the $I L-10$ rs $1800871 \mathrm{C} / \mathrm{T}$ genotype with improved survival in lowgrade glioma patients. $I L-10$ gene polymorphisms have been associated with multiple cancers, such as gastric [18], breast [19], and non-small cell lung cancer [20], as well as gliomas [9, 21]. Inflammation plays roles in all phases of tumor development, and IL-10, a well-known immuno-modulatory cytokine, may provide a functional link between inflammation and cancer [22]. Acuner-
Ozbabacan, et al. [22] found that IL-10 deficiency allowed for pro-inflammatory cytokine induction and hindered anti-tumor immunity, thereby promoting tumor growth. Tanikawa, et al. [23] observed that high serum IL-10 levels enhanced the tumor-specific immune response and reduced tumor growth. These results were inconsistent with earlier findings by Kim, et al. [24], who proposed that IL-10 inhibition would promote the anti-tumor immune response. These contrary findings may be due to the intrinsically pleiotropic biological activity of IL-10 and the variability of cancer models [25], or possibly genetic factors between 
Table 4: Univariate analysis of the impact of clinical factors on high-grade glioma patient OS and PFS

\begin{tabular}{|c|c|c|c|c|c|c|c|c|c|c|c|c|c|}
\hline \multirow{2}{*}{ Variable } & \multirow{2}{*}{ Classification } & \multirow{2}{*}{$\begin{array}{c}\text { No. of } \\
\text { patients/ } \\
\text { events }\end{array}$} & \multicolumn{4}{|c|}{ OS } & \multirow[b]{2}{*}{$p$} & \multicolumn{2}{|l|}{ No. of } & \multicolumn{3}{|c|}{ PFS } & \multirow[b]{2}{*}{$p$} \\
\hline & & & $\begin{array}{c}\text { 1/3-(year) } \\
\text { SR (\%) }\end{array}$ & MST & Log-rank $p$ & HR $(95 \%$ CI) & & $\begin{array}{c}\text { patients/ } \\
\text { events }\end{array}$ & $\begin{array}{c}\text { 1/3-(year) } \\
\text { SR (\%) }\end{array}$ & MST & Log-rank $p$ & HR (95\% CI) & \\
\hline \multirow[t]{2}{*}{ Gender } & Male & $107 / 102$ & $23.4 / 2.3$ & 11 & & 1 & & $106 / 105$ & $11.3 / 0.9$ & 8 & & 1 & \\
\hline & Female & $80 / 73$ & $33.7 / 8.4$ & 9 & 0.586 & $0.926(0.684-1.254)$ & 0.619 & $79 / 73$ & $16.5 / 7.4$ & 7 & 0.281 & $0.863(0.639-1.167)$ & 0.340 \\
\hline \multirow[t]{2}{*}{ Age(years) } & $<40$ & $64 / 59$ & $32.8 / 6.6$ & 10 & & 1 & & $63 / 60$ & $15.9 / 4.8$ & 8 & & 1 & \\
\hline & $\geq 40$ & $123 / 116$ & $25.2 / 5.0$ & 10 & 0.418 & $1.126(0.822-1.541)$ & 0.460 & $122 / 118$ & $12.3 / 3.1$ & 7 & 0.388 & $1.129(0.827-1.542)$ & 0.445 \\
\hline \multirow[t]{2}{*}{ WHO grade } & III & $124 / 116$ & $29.8 / 5.1$ & 11 & & 1 & & $123 / 118$ & $13.8 / 4.1$ & 8 & & 1 & \\
\hline & IV & $63 / 59$ & $23.8 / 6.3$ & 9 & 0.471 & $1.111(0.812-1.521)$ & 0.510 & $62 / 60$ & $12.9 / 3.2$ & 7 & 0.443 & $1.114(0.816-1.521)$ & 0.496 \\
\hline \multirow{2}{*}{ Extent of resection } & STR or NTR & $56 / 55$ & $16.1 / 1.8$ & 9 & & 1 & & $55 / 54$ & $1.8 / 1.8$ & 7 & & 1 & \\
\hline & GTR & $131 / 120$ & $32.8 / 6.9$ & 10 & 0.002 & $0.624(0.451-0.864)$ & 0.004 & $130 / 124$ & $18.5 / 4.5$ & 8 & 0.001 & $0.607(0.439-0.840)$ & 0.003 \\
\hline \multirow[t]{3}{*}{ Radiotherapy } & No & $20 / 18$ & $35.0 / 10.0$ & 8 & & 1 & & $19 / 19$ & $10.5 / 0.0$ & 6 & & 1 & \\
\hline & CRT & $49 / 42$ & $22.4 / 12.6$ & 9 & & $0.894(0.510-1.566)$ & 0.695 & $48 / 42$ & $18.8 / 12.5$ & 7 & & $1.005(0.579-1.744)$ & 0.987 \\
\hline & GK & $118 / 115$ & $28.8 / 2.5$ & 10 & 0.775 & $1.008(0.612-1.660)$ & 0.976 & $118 / 117$ & $11.9 / 0.8$ & 8 & 0.939 & $1.055(0.648-1.720)$ & 0.829 \\
\hline \multirow[t]{2}{*}{ Chemotherapy } & No & $113 / 110$ & 19.5/1.8 & 9 & & 1 & & $112 / 112$ & $12.5 / 3.6$ & 7 & & 1 & \\
\hline & Yes & $74 / 65$ & $40.5 / 11.3$ & 12 & 0.001 & $0.617(0.450-0.846)$ & 0.003 & $73 / 66$ & $15.1 / 9.4$ & 8 & 0.054 & $0.762(0.557-1.042)$ & 0.088 \\
\hline
\end{tabular}

OS: Overall survival; PFS: Progression free survival; WHO: World Health Organization; GTR: Gross-total resection; NTR: Near-total resection.

STR: Sub-total resection; CRT: Conformal radiation therapy; GK: Gamma knife; SR: Survival rate; MST: Median survival time (months).

HR: Hazard ratio; $95 \%$ CI: $95 \%$ Confidence interval.

Log-rank $p$ values were calculated from Chi-Square test.

$p$ values were calculated from Wald test.

$p<0.05$ indicates statistical significance.

Table 5: Univariate analysis of the association between rs1800871, rs7003908 and glioma patient OS and PFS

\begin{tabular}{|c|c|c|c|c|c|c|c|c|c|c|c|c|c|}
\hline \multirow{2}{*}{ SNP-ID } & \multirow{2}{*}{ Genotype } & \multirow{2}{*}{$\begin{array}{c}\text { No. of patients/ } \\
\text { events }\end{array}$} & \multicolumn{4}{|c|}{ os } & \multirow[b]{2}{*}{$p$} & \multirow{2}{*}{$\begin{array}{c}\text { No. of patients/ } \\
\text { events }\end{array}$} & \multicolumn{5}{|c|}{ PFS } \\
\hline & & & $\begin{array}{c}\text { 1/3-(year) } \\
\text { SR (\%) }\end{array}$ & MST & Log-rank $p$ & HR $(95 \%$ CI $)$ & & & $\begin{array}{c}\text { 1/3-(year) } \\
\text { SR (\%) }\end{array}$ & MST & Log-rank $p$ & HR $(95 \%$ CI $)$ & $p$ \\
\hline \multicolumn{14}{|c|}{ low-grade I-II } \\
\hline \multirow[t]{3}{*}{ rs 1800871} & $\mathrm{C} / \mathrm{C}$ & $36 / 35$ & $25.0 / 5.6$ & 10 & & 1 & & $36 / 35$ & $13.9 / 2.8$ & 7 & & 1 & \\
\hline & $\mathrm{C} / \mathrm{T}$ & $129 / 109$ & $39.5 / 13.1$ & 12 & & $0.745(0.573-0.969)$ & 0.028 & $129 / 118$ & $23.3 / 8.1$ & 8 & & $0.799(0.619-1.033)$ & 0.087 \\
\hline & $\mathrm{T} / \mathrm{T}$ & $126 / 116$ & $28.6 / 5.3$ & 11 & 0.019 & $1.098(0.752-1.603)$ & 0.627 & $124 / 119$ & $12.1 / 3.5$ & 8 & 0.079 & $1.087(0.745-1.585)$ & 0.665 \\
\hline \multirow[t]{3}{*}{ rs 7003908} & $\mathrm{~A} / \mathrm{A}$ & $188 / 168$ & $34.0 / 8.7$ & 12 & & 1 & & $186 / 176$ & $17.2 / 5.2$ & 8 & & 1 & \\
\hline & $\mathrm{C} / \mathrm{A}$ & $86 / 76$ & $32.6 / 11.4$ & 12 & & $0.965(0.736-1.265)$ & 0.796 & $86 / 80$ & $18.6 / 5.8$ & 8 & & $0.914(0.701-1.190)$ & 0.503 \\
\hline & $\mathrm{C} / \mathrm{C}$ & $20 / 19$ & $25.0 / 0.0$ & 12 & 0.843 & $1.105(0.687-1.777)$ & 0.68 & $20 / 19$ & $10.0 / 5.0$ & 9 & 0.755 & $0.956(0.595-1.536)$ & 0.852 \\
\hline \multicolumn{14}{|c|}{ high-grade III-IV } \\
\hline \multirow[t]{3}{*}{ rs 1800871} & $\mathrm{C} / \mathrm{C}$ & $22 / 20$ & $31.8 / 9.1$ & 11 & & 1 & & $22 / 20$ & $18.2 / 9.1$ & 8 & & 1 & \\
\hline & $\mathrm{C} / \mathrm{T}$ & $93 / 88$ & $24.7 / 4.3$ & 9 & & $1.133(0.824-1.557)$ & 0.444 & $91 / 89$ & $13.2 / 2.2$ & 7 & & $1.105(0.806-1.516)$ & 0.536 \\
\hline & $\mathrm{T} / \mathrm{T}$ & $71 / 67$ & $29.6 / 4.9$ & 10 & 0.467 & $0.883(0.536-1.455)$ & 0.626 & $71 / 68$ & $11.3 / 4.2$ & 8 & 0.598 & $0.908(0.551-1.496)$ & 0.704 \\
\hline \multirow[t]{3}{*}{ rs7003908 } & $\mathrm{A} / \mathrm{A}$ & $112 / 106$ & $32.1 / 3.1$ & 10 & & 1 & & $110 / 107$ & $13.6 / 2.7$ & 8 & & 1 & \\
\hline & $\mathrm{C} / \mathrm{A}$ & $69 / 63$ & $23.2 / 8.7$ & 10 & & $0.935(0.684-1.279)$ & 0.676 & $69 / 65$ & $14.5 / 5.8$ & 8 & & $0.979(0.718-1.333)$ & 0.891 \\
\hline & $\mathrm{C} / \mathrm{C}$ & $6 / 6$ & $0.0 / 0.0$ & 6 & 0.000 & $4.556(1.936-10.721)$ & 0.001 & $6 / 6$ & $0.0 / 0.0$ & 4 & 0.000 & $4.430(1.884-10.416)$ & 0.001 \\
\hline
\end{tabular}

SR: Survival rate; MST: Median survival time (months).

HR: Hazard ratio; $95 \%$ CI: $95 \%$ Confidence interval.

Log-rank $p$ values were calculated using the Chi-Square test.

$p$ values were calculated using the Wald test.

$p<0.05$ indicates statistical significance.

different races. The exact function of IL-10 in glioma is not clear; further studies are required to elucidate the mechanisms underlying the association between IL-10 polymorphisms and glioma patient prognosis.

This study was also the first to demonstrate that the PRKDC rs7003908 C/C genotype was correlated with poor prognosis in high-grade glioma patients. Rs7003908 was previously associated with increased risk of glioblastoma multiforme [14], hepatocellular carcinoma [26], prostate cancer [27], and bladder cancer [28]. PRKDC encodes the catalytic subunit of DNA-dependent protein kinase (DNA-PKcs), which is critical in the DNA damage response (DDR) and maintenance of genomic stability [29]. Goodwin, et al. [30] suggested that DNA-PKcs suppression inhibited tumor metastasis. Their group observed that DNA-PKcs induced cell migration, invasion, 
Table 6: Multivariate analysis of the association between rs1800871, rs7003908 and glioma patient OS and PFS

\begin{tabular}{|c|c|c|c|c|c|}
\hline \multirow{2}{*}{ SNP-ID } & \multirow{2}{*}{ Genotype } & \multicolumn{2}{|l|}{ OS } & \multicolumn{2}{|l|}{ PFS } \\
\hline & & Adjusted HR (95\% CI) & $p$ & Adjusted HR (95\% CI) & $p$ \\
\hline \multicolumn{6}{|l|}{ low-grade I-II } \\
\hline \multirow[t]{3}{*}{ rs 1800871} & $\mathrm{C} / \mathrm{C}$ & 1 & & 1 & \\
\hline & $\mathrm{C} / \mathrm{T}$ & $0.736(0.565-0.958)$ & 0.022 & $0.789(0.610-1.020)$ & 0.071 \\
\hline & $\mathrm{T} / \mathrm{T}$ & $1.073(0.735-1.567)$ & 0.716 & $1.059(0.726-1.545)$ & 0.767 \\
\hline \multirow[t]{3}{*}{ rs 7003908} & $\mathrm{~A} / \mathrm{A}$ & 1 & & 1 & \\
\hline & $\mathrm{C} / \mathrm{A}$ & $0.968(0.738-1.270)$ & 0.817 & $0.918(0.705-1.196)$ & 0.527 \\
\hline & $\mathrm{C} / \mathrm{C}$ & $1.119(0.696-1.799)$ & 0.643 & $0.962(0.599-1.547)$ & 0.874 \\
\hline \multicolumn{6}{|c|}{ high-grade III-IV } \\
\hline \multirow[t]{3}{*}{ rs 1800871} & $\mathrm{C} / \mathrm{C}$ & 1 & & 1 & \\
\hline & $\mathrm{C} / \mathrm{T}$ & $1.149(0.832-1.588)$ & 0.399 & $1.169(0.850-1.659)$ & 0.336 \\
\hline & $\mathrm{T} / \mathrm{T}$ & $0.879(0.533-1.449)$ & 0.612 & $0.929(0.564-1.532)$ & 0.774 \\
\hline \multirow[t]{3}{*}{ rs7003908 } & $\mathrm{A} / \mathrm{A}$ & 1 & & 1 & \\
\hline & $\mathrm{C} / \mathrm{A}$ & $1.038(0.755-1.427)$ & 0.82 & $1.033(0.756-1.412)$ & 0.839 \\
\hline & $\mathrm{C} / \mathrm{C}$ & $4.288(1.808-10.169)$ & 0.001 & $3.783(1.601-8.943)$ & 0.002 \\
\hline
\end{tabular}

OS: Overall survival; PFS: Progression free survival.

HR: Hazard ratio; 95\% CI: 95\% Confidence interval.

$p$ values were calculated using the Wald test.

$p<0.05$ indicates statistical significance.

and metastasis, and was highly activated in advanced tumors, independent of DNA damage indicators. The same group [29] showed that PRKDC, as a transcriptional regulator, might promote tumor initiation and progression. Additionally, $P R K D C$ expression and activity is increased in numerous tumor types, such as colorectal cancer [31], esophageal cancer [10], and B-cell chronic lymphocytic leukemias [11]. In the present study, we observed reduced $\mathrm{OS}$ and PFS in high-grade glioma patients carrying the $P R K D C$ rs $7003908 \mathrm{C} / \mathrm{C}$ genotype. This mutation may be associated with increased $P R K D C$ expression, activity, or receptor activation, any of which might promote cell proliferation.

In conclusion, our data indicated that chemotherapy and extent of tumor resection were associated with improved glioma patient prognosis. IL-10 SNP rs 1800871 was associated with improved survival in low-grade glioma cases, while PRKDC SNP rs7003908 was correlated with poor prognosis in high-grade patients. Although the exact mechanisms remain unclear, our results suggest that these two SNPs may be useful biomarkers for predicting glioma patient outcomes. Additionally, IL-10 and DNA-PKcs may be promising therapeutic targets for treatment of advanced malignancies. Further functional studies are needed to evaluate associations between rs1800871 and rs7003908 polymorphisms and glioma patient prognosis.

\section{MATERIALS AND METHODS}

\section{Subjects}

A total of 481 patients diagnosed with glioma at the Department of Neurosurgery, Tangdu Hospital, Fourth Military Medical University, Shaanxi Province (Xi'an, China) between September 2010 and April 2016 were randomly enrolled in this study. We used the following detailed selection criteria: recently diagnosed and histologically confirmed to have glioma (glioma was diagnosed via imaging and pathological analysis); Han Chinese patient with no kinship; underwent regular follow-up; peripheral blood samples available; no previous history of other cancers; and no prior treatment for glioma or prior treatment with chemotherapy or radiotherapy. Histopathological diagnosis was confirmed according to the World Health Organization (WHO) classification in 2007.

Our study protocol adhered to the principles of the Declaration of Helsinki and was reviewed and approved by the Ethics Committee of Tangdu Hospital. All patients gave written informed consent prior to participation in the study.

\section{DNA extraction and genotyping}

Genomic DNA was extracted from glioma patient peripheral blood samples (5 mL) using GoldMagMini Whole Blood Genomic DNA Purification Kits 
(GoldMag. Co. Ltd., Xi'an, China) according to the manufacturer's protocols. DNA quantity was evaluated by spectrophotometry (DU530UV/VIS spectrophotometer, Beckman Instruments, Fullerton, CA, USA). We designed polymerase chain reaction (PCR) and extension primers for the SNPs using the Sequenom MassARRAY Assay Design 3.0 software (Sequenom, San Diego, CA, USA). Sequenom MassARRAY RS1000 was utilized to genotype SNPs according to the manufacturer's protocol. We used Sequenom Typer 4.0 software for data management and analysis.

\section{Clinical data and follow-up}

Patient treatment and survival (overall and progression-free) information was collected from a retrospective review of patient medical records or consultation with treating physicians. A standardized questionnaire was used to collect clinical data, including the date of diagnosis, follow-up date(s), patient age and gender, tumor histologic type, extent of resection, exact pathology, WHO grade, surgical methods, and postoperative radiotherapy and chemotherapy regimen(s). Follow-up consisted of outpatient visits, telephone interviews, and written communication with patients or their families. Clinical follow-up was performed in singleblind fashion with death as the end point. Glioma patients who were alive at the end of the follow-up period were excluded from the study. Data were stored electronically using the EpiData3.02 software, and validation, revision and conversion of assigned values were performed to establish the glioma patient database used for analysis.

\section{Statistical analyses}

SPSS 17.0 (SPSS, Chicago, IL, USA) was used to analyze follow-up and experimental data. Log-rank tests were used to compare survival curves. Univariate and multivariable Cox proportional hazard models were used to calculate crude and adjusted hazard ratios (HRs) and 95\% confidence intervals (CIs), respectively. Two-sided $p$-values $<0.05$ were considered statistically significant and were calculated using the Wald test. HRs were adjusted for other factors that could affect glioma outcome, such as patient age and gender, and extent of tumor resection.

\section{ACKNOWLEDGMENTS}

It is our great honor to express heartfelt thanks to all of the patients, clinicians and other hospital staff who participated in and contributed to this study.

\section{CONFLICTS OF INTEREST}

The authors declare no conflicts of interest.

\section{GRANT SUPPORT}

This work was supported by This work was supported by Science and Technology Program of Xi'an (No. SF1510(5)).

\section{REFERENCES}

1. Wrensch M, Minn Y, Chew T, Bondy M, Berger MS. Epidemiology of primary brain tumors: current concepts and review of the literature. Neuro-oncology. 2002; 4:278-299.

2. Louis DN, Ohgaki H, Wiestler OD, Cavenee WK, Burger PC, Jouvet A, Scheithauer BW, Kleihues P. The 2007 WHO classification of tumours of the central nervous system. Acta neuropathologica. 2007; 114:97-109.

3. Louis DN, Holland EC, Cairncross JG. Glioma classification: a molecular reappraisal. Am J Pathol. 2001; 159:779-786.

4. Lamborn KR, Yung WK, Chang SM, Wen PY, Cloughesy TF, DeAngelis LM, Robins HI, Lieberman FS, Fine HA, Fink KL, Junck L, Abrey L, Gilbert MR, et al. Progression-free survival: an important end point in evaluating therapy for recurrent high-grade gliomas. Neurooncology. 2008; 10:162-170.

5. Wrensch M, Fisher JL, Schwartzbaum JA, Bondy M, Berger M, Aldape KD. The molecular epidemiology of gliomas in adults. Neurosurgical focus. 2005; 19:E5.

6. Haque A, Banik NL, Ray SK. Molecular alterations in glioblastoma: potential targets for immunotherapy. Prog Mol Biol Trans. 2011; 98:187-234.

7. Xue H, Lin B, An J, Zhu Y, Huang G. Interleukin-10-819 promoter polymorphism in association with gastric cancer risk. BMC cancer. 2012; 12:102.

8. Huettner C, Czub S, Kerkau S, Roggendorf W, Tonn JC. Interleukin 10 is expressed in human gliomas in vivo and increases glioma cell proliferation and motility in vitro. Anticancer Res. 1997; 17:3217-3224.

9. Jin T, Li X, Zhang J, Wang H, Geng T, Li G, Gao G, Chen C. Genetic association between selected cytokine genes and glioblastoma in the Han Chinese population. BMC cancer. 2013; 13:236.

10. Tonotsuka N, Hosoi Y, Miyazaki S, Miyata G, Sugawara K, Mori T, Ouchi N, Satomi S, Matsumoto Y, Nakagawa K, Miyagawa K, Ono T. Heterogeneous expression of DNA-dependent protein kinase in esophageal cancer and normal epithelium. Int J Mol Med. 2006; 18:441-447.

11. Willmore E, Elliott SL, Mainou-Fowler T, Summerfield GP, Jackson GH, O’Neill F, Lowe C, Carter A, Harris R, Pettitt AR, Cano-Soumillac C, Griffin RJ, Cowell IG, et al. DNA-dependent protein kinase is a therapeutic target and an indicator of poor prognosis in B-cell chronic lymphocytic leukemia. Clin Cancer Res. 2008; 14:3984-3992. 
12. Sun S, Cheng S, Zhu Y, Zhang P, Liu N, Xu T, Sun C, Lv Y. Identification of PRKDC (Protein Kinase, DNA-Activated, Catalytic Polypeptide) as an essential gene for colorectal cancer (CRCs) cells. Gene. 2016; 584:90-96.

13. van Duin $\mathrm{M}$, Koken $\mathrm{MH}$, van den Tol J, ten Dijke P, Odijk H, Westerveld A, Bootsma D, Hoeijmakers JH. Genomic characterization of the human DNA excision repair gene ERCC-1. Nucleic Acids Res. 1987; 15:91959213.

14. McKean-Cowdin R, Barnholtz-Sloan J, Inskip PD, Ruder AM, Butler M, Rajaraman P, Razavi P, Patoka J, Wiencke JK, Bondy ML, Wrensch M. Associations between polymorphisms in DNA repair genes and glioblastoma. Cancer epidemiology, biomarkers \& prevention. 2009; 18:1118-1126.

15. Li B, Zhao W, Li J, Yan M, Xie Z, Zhu Y, Chen C, Jin T. Effect of epidermal growth factor receptor gene polymorphisms on prognosis in glioma patients. Oncotarget. 2016; 7:63054 63064. doi: 10.18632/oncotarget.10666.

16. Sanai N, Berger MS. Extent of resection influences outcomes for patients with gliomas. Rev Neurol-France. 2011; 167:648-654.

17. Stewart LA. Chemotherapy in adult high-grade glioma: a systematic review and meta-analysis of individual patient data from 12 randomised trials. Lancet (London, England). 2002; 359:1011-1018.

18. Yuan LJ, Jin TB, Yin JK, Du XL, Wang Q, Dong R, Wang SZ, Cui Y, Chen C, Lu JG. Polymorphisms of tumor-related genes IL-10, PSCA, MTRR and NOC3L are associated with the risk of gastric cancer in the Chinese Han population. Cancer Epidemiol. 2012; 36:e366-372.

19. Pooja S, Chaudhary P, Nayak LV, Rajender S, Saini KS, Deol D, Kumar S, Bid HK, Konwar R. Polymorphic variations in IL-1beta, IL-6 and IL-10 genes, their circulating serum levels and breast cancer risk in Indian women. Cytokine. 2012; 60:122-128.

20. Shih CM, Lee YL, Chiou HL, Hsu WF, Chen WE, Chou MC, Lin LY. The involvement of genetic polymorphism of IL-10 promoter in non-small cell lung cancer. Lung cancer (Amsterdam, Netherlands). 2005; 50:291-297.

21. Shamran HA, Ghazi HF, Al-Salman A, Al-Juboory AA, Taub DD, Price RL, Nagarkatti M, Nagarkatti PS, Singh UP. Single Nucleotide Polymorphisms in IL-10, IL-12p40, and
IL-13 Genes and Susceptibility to Glioma. Int J Med Sci. 2015; 12:790-796.

22. Acuner-Ozbabacan ES, Engin BH, Guven-Maiorov E, Kuzu G, Muratcioglu S, Baspinar A, Chen Z, Van Waes C, Gursoy A, Keskin O, Nussinov R. The structural network of Interleukin-10 and its implications in inflammation and cancer. BMC genomics. 2014; 15:S2.

23. Tanikawa T, Wilke CM, Kryczek I, Chen GY, Kao J, Nunez G, Zou W. Interleukin-10 ablation promotes tumor development, growth, and metastasis. Cancer research. 2012; 72:420-429.

24. Kim BG, Joo HG, Chung IS, Chung HY, Woo HJ, Yun YS. Inhibition of interleukin-10 (IL-10) production from MOPC 315 tumor cells by IL-10 antisense oligodeoxynucleotides enhances cell-mediated immune responses. Cancer immunology, immunotherapy. 2000; 49:433-440.

25. Mocellin S, Marincola FM, Young HA. Interleukin-10 and the immune response against cancer: a counterpoint. J Leukocyte Biol. 2005; 78:1043-1051.

26. Yao JG, Huang XY, Long XD. Interaction of DNA repair gene polymorphisms and aflatoxin B1 in the risk of hepatocellular carcinoma. Int J Clin Exp Patho. 2014; 7:6231-6244.

27. Mandal RK, Kapoor R, Mittal RD. Polymorphic variants of DNA repair gene $\mathrm{XRCC} 3$ and $\mathrm{XRCC} 7$ and risk of prostate cancer: a study from North Indian population. DNA Cell Biol. 2010; 29:669-674.

28. Gangwar R, Ahirwar D, Mandhani A, Mittal RD. Do DNA repair genes OGG1, XRCC3 and XRCC7 have an impact on susceptibility to bladder cancer in the North Indian population? Mutation research. 2009; 680:56-63.

29. Goodwin JF, Knudsen KE. Beyond DNA repair: DNA-PK function in cancer. Cancer Discov. 2014; 4:1126-1139.

30. Goodwin JF, Kothari V, Drake JM, Zhao S, Dylgjeri E, Dean JL, Schiewer MJ, McNair C, Jones JK, Aytes A, Magee MS, Snook AE, Zhu Z, et al. DNA-PKcs-Mediated Transcriptional Regulation Drives Prostate Cancer Progression and Metastasis. Cancer cell. 2015; 28:97-113.

31. Hosoi Y, Watanabe T, Nakagawa K, Matsumoto Y, Enomoto A, Morita A, Nagawa H, Suzuki N. Up-regulation of DNAdependent protein kinase activity and $\mathrm{Sp} 1$ in colorectal cancer. Int J Oncol. 2004; 25:461-468. 